\title{
EFEITO DE FUNGOS ANTAGONISTAS E PRODUTOS QUÍMICOS NO CONTROLE DA PODRIDÃO PARDA EM POMARES DE PESSEGUEIRO
}

\author{
Luciene Martins Moreira*, Louise L. May-De Mio* \\ *Eng ${ }^{\mathrm{a}}$. Agrônoma, Dr ${ }^{\mathrm{a}}$., Depto. de Fitotecnia e Fitossanitarismo, UFPR - lmmoreira@terra.com.br; maydemio@ufpr.br
}

Recebido para publicação: 21/02/2006 - Aceito para publicação: 04/07/2006

\begin{abstract}
Resumo
Um experimento com controle biológico em campo foi conduzido no município da Lapa-PR, para o controle de Monilinia fructicola em pessegueiro cultivar BR-1. Agentes biológicos previamente selecionados de campo de produção foram comparados com o fungicida iprodione e com fosfitos de $\mathrm{Ca}$ e de K. O experimento contou com 10 tratamentos: seis fungos antagonistas (F1, F2 e F4 Trichothecium roseum; F9 - Penicillium sp; F3 - Cephalosporium sp; F13 - Alternaria sp), dois fosfitos $(\mathrm{Ca}$ e $\mathrm{K})$, iprodione e testemunha. A avaliação foi feita por incidência no campo e na póscolheita, sendo os tratamentos aplicados por pulverização e imersão, respectivamente. Em relação à incidência em campo, destacaram-se o iprodione e, na seqüência, os fosfitos de $\mathrm{K}$, com $71,4 \%$, e de $\mathrm{Ca}$, com $64,2 \%$ de controle. Entre os antagonistas, destacaram-se F3, F1 e F2, com nível de controle superior a $64 \%$ em relação à testemunha. Após a colheita, os frutos foram mantidos à temperatura ambiente e avaliados no terceiro dia de exposição pela incidência da doença. Nesse momento, foi observada uma baixa eficiência entre os tratamentos, somente destacando-se o fungicida. Também foram avaliados o peso médio dos frutos, diâmetro, firmeza da polpa e sólidos solúveis totais, não havendo diferença estatística entre os tratamentos. Paralelamente às avaliações de campo, frutos não pulverizados foram colhidos para receberem em pós-colheita os mesmos tratamentos executados no campo. Estes foram feridos, imersos nos tratamentos e avaliados pela incidência da doença no segundo e quarto dias de exposição no ambiente. Iprodione, fosfito de Ca e F1 exerceram o maior controle das infecções latentes. Nas áreas feridas, destacaram-se iprodione, F2 e F4.

Palavras-chave: Monilinia fructicola; controle biológico; pré-colheita; pós-colheita; fosfito.
\end{abstract}

\begin{abstract}
Effect of antagonist fungi and chemical products in the control of brown rot in peach orchards. An experiment with biological control in the field was conducted in the county of Lapa-PR, for the control of Monilinia fructicola in peach cultivar BR-1. Biological agents previously selected from the production field were compared with the fungicide iprodione and with $\mathrm{Ca}$ and $\mathrm{K}$ phosphites. The experiment consisted of 10 treatments, being six antagonist fungi (F1, F2 and F4 - Trichothecium roseum; F9 - Penicillium sp; F3 Cephalosporium sp; F13 - Alternaria sp), two phosphites (Ca and $\mathrm{K}$ ), iprodione and check. The evaluation was carried out per field incidence and postharvest and the treatments were applied by means of pulverization and immersion, respectively. In relation to the field incidence, the following could be pointed out: iprodione, and in sequence, $\mathrm{K}$ phosphites with 71,4 and Ca phosphites with $64,2 \%$ control, and among the antagonist fungi F3, F1 and F2 with control level superior to $64 \%$ in relation to the check. After harvest, the fruits were kept at room temperature and evaluated on the third day of exposure by disease incidence. At this moment low efficiency among the treatments was observed, exception only to the fungicide. The following were also evaluated: the average weight of the fruits, diameter, pulp firmness and total soluble solids, and no statistical difference between the treatments was observed. Parallel to the field evaluation, the non-pulverized fruits were harvested to receive, in postharvest, the same treatments carried out in the field. They were damaged, immersed into the treatments and evaluated according to the incidence of disease on the second and fourth exposure days in the environment. Iprodione, Ca phosphite and F1 exerted higher control of the latent infections. In the damaged areas, the action of iprodione and F2 and F4 were most noticeable.
\end{abstract}

Keywords: Monilinia fructicola; biological control; preharvest; postharvest; phosphite. 


\section{INTRODUÇÃO}

A podridão parda do pessegueiro (Prunus persica (L.) Batsch), causada pelo fungo Monilinia fructicola (Wint) Honey, é a principal doença dessa cultura. A infecção inicia nas flores e passa para os ramos, os quais podem fornecer inóculo para os frutos durante o crescimento e na maturação (HONG et al. 1998; LARENA et al. 2005).

Algumas práticas auxiliam no controle da doença, como a poda de ramos doentes e a eliminação de frutos mumificados remanescentes, visando reduzir o inóculo no período de amadurecimento dos frutos.

O uso de fungicidas é o método mais adotado para controlar a podridão parda, e segundo MayDe Mio et al. (2004) podem ser utilizados captan, mancozeb, iprodione e tebuconazole, além de procimidone, difeconazole, fluazinam e chlorothalonil (WADT et al. 1999). Entretanto, há razões que restringem o uso desses produtos, tais como a necessidade de redução do uso de fungicidas próximo e após a colheita dos frutos (IPPOLITO; NIGRO, 2000). Como método alternativo, há a utilização de microrganismos que ocorrem naturalmente na superfície das plantas e têm sido propostos como antagonistas a $M$. fructicola. No controle desse patógeno, já foram selecionados Penicillium frequentans Westling, Epicoccum nigrum Link, Trichothecium roseum (Pers.:Fr.) Link, Aureobasidium pullulans (de Bary) Arnaud, E. purpurascens Ehrenberg, Gliocladium roseum Bainier (DE CAL et al. 1990; HONG; MICHAILIDES, 1997; MADRIGAL et al. 1994; WITTIG et al. 1997).

Diversas pesquisas reportam o uso do controle biológico em pós-colheita (BONATERRA et al. 2003; SCHENA et al. 2003), pois se acredita que em ambientes onde as condições são controladas, os agentes seriam mais facilmente aplicados (IPPOLITO; NIGRO, 2000). Porém, há também relatos do emprego de antagonistas durante a pré-colheita, na tentativa de desenvolver um método alternativo para o controle da podridão, como, por exemplo, pulverizações de $P$. frequentans e $E$. nigrum em pessegueiro e A. pullulans e E. purpurascens em cerejeira (Prunus avium L.) (DE CAL et al. 1990; MADRIGAL et al. 1994; WITTIG et al. 1997). Apesar dos trabalhos realizados, faltam pesquisas com controle da podridão parda pela associação de antagonistas a outros produtos, como fosfitos, o que justifica a realização deste trabalho. Para tanto, objetivou-se avaliar o controle da doença no campo e em pós-colheita com antagonistas, fungicidas e fosfitos de $\mathrm{Ca}$ e $\mathrm{K}$.

\section{MATERIAL E MÉTODOS}

\section{Obtenção dos isolados dos antagonistas}

Os isolados foram obtidos de frutos infectados ou mumificados de pessegueiro e ameixeira (Prunus salicina Lindl.) de diversas cultivares, em pomares comerciais do município da Lapa/PR (Tabela 1). Esses fungos, que cresciam sobre ou próximos às lesões do patógeno, foram isolados em meio de cultura BDA (batata-dextrose-ágar), codificados em F1, F2, F4 (T. roseum), F9 (Penicillium sp), F3 (Cephalosporium sp) e F13 (Alternaria sp) e utilizados como tratamentos visando o antagonismo a M. fructicola.

Tabela 1. Isolados dos fungos antagonistas obtidos de frutos coletados na Lapa/PR.

Table 1. Solates of antagonistics fungi obtained from fruits collected at Lapa/PR.

\begin{tabular}{ccc}
\hline Isolado $^{1}$ & Planta & Cultivar \\
\hline F1 & Ameixa & H.P. (Harry Pickstone) \\
F2 & Pêssego & BR-1 \\
F3 & Pêssego & BR-1 \\
F4 & Pêssego & Chimarrita \\
F9 & Pêssego & Ouro \\
F13 & Pêssego & Chimarrita \\
\hline${ }^{1}$ F1, F2 e F4 = Trichothecium roseum $;$ F9 = Penicillium sp; F3 = Cephalosporium sp; F13 = Alternaria $\mathrm{sp}$.
\end{tabular}

\section{Controle da podridão parda em campo}

O experimento foi conduzido com a cultivar BR-1, no município da Lapa/PR, em delineamento inteiramente casualizado com seis repetições. A unidade experimental constituiu-se de dois ramos marcados em cada lateral das plantas. Foram utilizados 10 tratamentos, seis com agentes biológicos (F1, F2, F4 - $10^{6}$ esporos/mL; F3, F9, F13 - $10^{7}$ esporos $\left./ \mathrm{mL}\right)$, dois fosfitos $\left(\mathrm{Ca}-6 \%\right.$ e K - $\mathrm{K}_{2} 020 \%$ a $200 \mathrm{~mL} / 100$ $\mathrm{L}$ de água); iprodione (150 mL p.c.) e testemunha. Os tratamentos foram aspergidos em intervalo de 13 dias (10 
aplicações), por meio de pulverizadores manuais com capacidade para $500 \mathrm{~mL}$. A produção de propágulos dos microrganismos foi feita em placas de Petri com meio de cultura BDA colonizado pelos fungos (10 placas/agente). Para o preparo das suspensões, foi adicionada água às culturas e às estruturas suspensas com auxílio de pincel, sendo a seguir filtradas em tecido de algodão. Foi realizada avaliação da doença no campo, e foram colhidos 10 frutos (parcela experimental), avaliando-se o peso médio e diâmetro. Em quatro deles foi feita a determinação da firmeza da polpa (penetrômetro - marca TR, modelo FT327) e dos sólidos solúveis totais (refratômetro - marca Huake, modelo RHB-32atc). Os seis frutos restantes foram colocados em bandejas à temperatura ambiente $\left(24^{\circ} \mathrm{C}\right)$ e após três dias foi registrada a incidência da doença. As análises foram executadas com programa SASM-Agri versão 8.0 (CANTERI et al. 2001), utilizando-se o teste de Duncan a $5 \%$ para comparação de médias e o teste de Scott-Knott a 5\% para os parâmetros de qualidade dos frutos.

\section{Avaliação do controle químico e biológico da podridão parda do pessegueiro, em pós-colheita}

Neste experimento, pêssegos colhidos nas mesmas plantas do ensaio em pré-colheita, entretanto não pulverizados, receberam em pós-colheita os mesmos tratamentos avaliados em campo. Os frutos receberam quatro ferimentos, com estilete flambado, na região oposta ao pedúnculo. Em seguida, foram imersos nos diferentes tratamentos durante um minuto. A testemunha constituiu-se da imersão dos frutos em água esterilizada. Em seguida, foram dispostos em bandejas plásticas, à temperatura ambiente de aproximadamente $28{ }^{\circ} \mathrm{C}$. A avaliação da incidência da doença foi feita no segundo e quarto dias após a aplicação dos tratamentos, observando-se o surgimento de infecções fora dos ferimentos (infecção latente) e na região dos ferimentos. As análises dos resultados foram feitas para delineamento inteiramente casualizado para 10 tratamentos e três repetições pelo programa SASM-Agri versão 8.0 (CANTERI et al. 2001), utilizando-se o teste de Scott-Knott a 5\% para comparação de médias.

\section{RESULTADOS E DISCUSSÃO}

\section{Avaliação do controle químico e biológico da podridão parda do pessegueiro, em pré-colheita}

Nos frutos avaliados no campo, a incidência foi baixa. Somente foi verificada diferença significativa pelo teste de Duncan a $5 \%$ no tratamento com iprodione (Tabela 2). Nos frutos mantidos até três dias no ambiente, verificou-se baixa eficiência dos tratamentos, com diferença significativa entre o tratamento iprodione e os demais, tendo aquele apresentado $14 \%$ de frutos doentes, enquanto $\mathrm{F} 2$, fosfito de Ca, F4 e F9 não controlaram a doença quando comparados à testemunha (Tabela 2). A elevada incidência da doença nessa fase comprova que, na colheita e pós-colheita, o patógeno se expressa com mais intensidade, pois nesse período há condições de temperatura e umidade ideais à esporulação do fungo (BONATERRA et al. 2003; HONG et al. 1998; LUO; MICHAILIDES, 2003; WITTIG et al. 1997).

Tabela 2. Porcentagem de podridão parda do pessegueiro em frutos tratados durante a pré-colheita com ingredientes ativos químicos e agentes de controle biológico.

Table 2. Peach brown rot percentage in fruits treated during preharvest with chemical products and biological agents.

\begin{tabular}{|c|c|c|c|c|}
\hline \multirow{2}{*}{ Tratamentos } & \multicolumn{4}{|c|}{ Porcentagem de podridão parda ${ }^{1}$} \\
\hline & \multicolumn{2}{|c|}{ No campo $^{2}$} & \multicolumn{2}{|c|}{ Aos 3 dias } \\
\hline T. roseum $(\mathrm{F} 1)$ & 9 & $\mathrm{ab}$ & 64 & $\mathrm{ab}$ \\
\hline T. roseum $(\mathrm{F} 2)$ & 9 & $\mathrm{ab}$ & 94 & $\mathrm{a}$ \\
\hline Cephalosporium sp (F3) & 3 & $\mathrm{ab}$ & 64 & $a b$ \\
\hline T. roseum $(\mathrm{F} 4)$ & 13 & $\mathrm{a}$ & 83 & $\mathrm{ab}$ \\
\hline Penicillium sp (F9) & 13 & $\mathrm{a}$ & 75 & $a b$ \\
\hline Alternaria sp (F13) & 10 & $\mathrm{ab}$ & 64 & $\mathrm{~b}$ \\
\hline Fosfito $\mathrm{Ca}(\mathrm{Ca} 6 \%)$ & 5 & $\mathrm{ab}$ & 86 & $a b$ \\
\hline Fosfito $\mathrm{K}\left(\mathrm{K}_{2} \mathrm{O} 20 \%\right)$ & 4 & $\mathrm{ab}$ & 56 & $\mathrm{~b}$ \\
\hline Iprodione & 0 & $\mathrm{~b}$ & 14 & $\mathrm{c}$ \\
\hline Testemunha & 14 & $\mathrm{a}$ & 75 & $a b$ \\
\hline $\mathrm{CV}(\%)$ & \multicolumn{2}{|c|}{94,23} & \multicolumn{2}{|c|}{39,74} \\
\hline
\end{tabular}


$\mathrm{O}$ fosfito de $\mathrm{K}$ proporcionou $25,3 \%$ de controle, mostrando-se mais efetivo que o de $\mathrm{Ca}$, conforme os resultados obtidos por Moreira (1999), que, ao pulverizá-los em pré-colheita, teve $41 \%$ dos frutos com podridão contra $70,6 \%$ com o fosfito de Ca no mesmo intervalo de avaliação. $\mathrm{O}$ uso dos fosfitos tem despertado interesse nas pesquisas, pois se apresentam como uma alternativa aos fungicidas, contribuindo para que seja evitada a resistência. Com eles busca-se uma atuação quanto à indução de substâncias de autodefesa, as fitoalexinas, sendo empregados para o controle de doenças em pessegueiro - contra M. fructicola (MOREIRA, 1999) -, em macieira (Malus domestica Borkh) - contra Phytophthora spp. Butler, Venturia inaequalis (Cooke) Winter e Colletotrichum spp. (Penz.) (BONETI; KATSURAYAMA, 2002) -, e em videira (Vitis vinifera L.) - contra Plasmopara viticola (Berk. et Curtis) (DALBÓ; SCHUCK, 2003; SÔNEGO et al. 2003).

Os antagonistas que exerceram algum controle foram F1, F3 e F13, com 14,7\% contra 75\% de doença apresentada pela testemunha. Apesar do desempenho dos controladores biológicos neste trabalho, o fungo $P$. frequentans foi utilizado em outros experimentos em pré e pós-colheita, proporcionando até $80 \%$ de controle da podridão (DE CAL et al. 1990). Os antagonistas utilizados neste trabalho não apresentaram colonização dos frutos nem na avaliação de campo nem na de pós-colheita, sendo assim potenciais para controle. Portanto devem ser testados em outras condições, principalmente em pulverização desde a fase de floração, com o intuito de deixar o antagonista se estabelecer no ambiente para a fase mais crítica de disseminação do patógeno, durante o amadurecimento dos frutos.

A escolha desses antagonistas foi a partir de um experimento realizado por Moreira (1999), quando eles foram utilizados para tratamento dos frutos em pós-colheita, apresentando bom desempenho e tornando-se candidatos a serem testados em campo. No ciclo em que se realizou este experimento, as aplicações iniciaram logo após a floração, o que pode justificar a elevada incidência de doença nos frutos avaliados em pós-colheita, aos três dias, podendo-se concluir que o controle biológico utilizado apenas na pré-colheita pode comprometer o desempenho do antagonista testado. Vários autores citam a importância da aplicação de tratamentos durante a floração contra Monilinia spp (EMERY et al. 2002; HONG et al. 1998; LUO et al. 2001).

Não houve diferença estatística nos parâmetros peso médio, diâmetro, firmeza da polpa e sólidos solúveis totais, entre os tratamentos, pelo teste de Scott-Knott a 5\%. O peso manteve-se em torno de 630 $\mathrm{g}$, o diâmetro em $5 \mathrm{~cm}$, a firmeza da polpa em 13 libras e o teor de sólidos solúveis totais ao redor de 10 ${ }^{\circ}$ Brix (Tabela 3).

Tabela 3. Médias dos parâmetros: sólidos solúveis totais ( ${ }^{\circ}$ Brix), peso médio, diâmetro e firmeza da polpa em pêssegos cultivar BR-1 tratados em pré-colheita.Lapa/PR.

Table 3. Average of parameters: total soluble solids ( $\left.{ }^{\circ} \mathrm{Brix}\right)$, fruit weight, diameter and pulp firmness on peach cultivar BR-1 treated in prehearvest. Lapa/PR.

\begin{tabular}{lcccc}
\hline \multicolumn{1}{c}{ Tratamentos } & $\begin{array}{c}\text { Graus } \\
\text { Brix }\end{array}$ & $\begin{array}{c}\text { Peso médio } \\
(\mathbf{g})\end{array}$ & $\begin{array}{c}\text { Diâmetro } \\
(\mathbf{c m})\end{array}$ & $\begin{array}{c}\text { Firmeza } \\
(\mathbf{l b})\end{array}$ \\
\hline T. roseum (F1) & $10,5 \mathrm{~ns}$ & $669,8 \mathrm{Ns}$ & $5,3 \mathrm{~ns}$ & $15,0 \mathrm{~ns}$ \\
T. roseum (F2) & 10,3 & 666,0 & 5,2 & 13,6 \\
Cephalosporium sp (F3) & 10,3 & 651,0 & 5,2 & 13,5 \\
T. roseum (F4) & 10,2 & 645,8 & 5,2 & 13,5 \\
Penicillium sp (F9) & 10,2 & 644,0 & 5,2 & 13,0 \\
Alternaria sp (F13) & 10,1 & 642,5 & 5,2 & 12,9 \\
Fosfito Ca (Ca 6\%) & 10,0 & 638,7 & 5,2 & 12,5 \\
Fosfito K (K $20 \%)$ & 9,6 & 626,7 & 5,1 & 12,1 \\
Iprodione & 9,6 & 606,7 & 5,1 & 12,0 \\
Testemunha & 9,5 & 606,3 & 5,1 & 11,8 \\
\hline CV (\%) & 8,11 & 11,92 & 4,19 & 15,68 \\
\hline Dados originais. As médias seguidas verticalmente pela mesma letra não diferem estatisticamente pelo teste de Scott-
\end{tabular}

Dados originais. As médias seguidas verticalmente pela mesma letra não diferem estatisticamente pelo teste de ScottKnott a 5\% de significância.

\section{Avaliação em pós-colheita do controle de Monilinia fructicola por produtos químicos e agentes biológicos}

$\mathrm{Na}$ avaliação referente às infecções latentes, destacaram-se os tratamentos iprodione e fosfito de $\mathrm{Ca}$, com 95 e 47,6\% de controle. Já entre os antagonistas, o melhor foi $\mathrm{F} 1$, com $25,4 \%$ de eficiência, enquanto a testemunha apresentou $63 \%$ de frutos com podridão no segundo dia de avaliação (Tabela 4). 
Aos quatro dias, somente o tratamento com iprodione manteve-se eficiente, e os demais chegaram próximo ou atingiram $100 \%$ de incidência.

Com relação à incidência da podridão nos ferimentos, os frutos tratados com iprodione e com os três isolados de T. roseum apresentaram a mais elevada porcentagem de controle: 100 (fungicida); 72,7 (F2); 62,3 (F4) e 54,5\% (F1), enquanto a testemunha teve 55\% de doença nas áreas feridas no segundo dia de avaliação (Tabela 4). No quarto dia, no ambiente, o iprodione permaneceu eficiente, com 96,8\% de controle, e os outros tratamentos não diferiram da testemunha, que exibiu $95 \%$ de podridão nos ferimentos.

Os fosfitos não controlaram a podridão parda neste experimento, sendo que somente o de $\mathrm{Ca}$ diferiu da testemunha e dos demais tratamentos aos 2 dias de avaliação no controle das infecções latentes. Aos quatro dias esse efeito diminuiu, não havendo diferença com relação à testemunha (Tabela 4). Para Moreira et al. (2002), os isolados de T. roseum foram mais efetivos, e o fosfito de K mostrou-se melhor tanto nas avaliações de controle das infecções latentes quanto nos ferimentos.

Tabela 4. Porcentagem de infecções latentes e média de ferimentos com podridão parda em pêssegos tratados em pós-colheita com ingredientes ativos químicos e agentes de controle biológico. Lapa/PR.

Table 4. Percentage of latent infection and average of wounds with peach brown rot treated in post harvest with chemicals and biological agents. Lapa/PR.

\begin{tabular}{|c|c|c|c|c|}
\hline \multirow{3}{*}{ Tratamentos } & \multicolumn{4}{|c|}{ Tratamento pós-colheita ${ }^{1}$} \\
\hline & \multicolumn{2}{|c|}{ Lesões latentes $(\%)^{2}$} & \multicolumn{2}{|c|}{ Lesões nos ferimentos $(\%)^{2}$} \\
\hline & 2 dias & 4 dias & 2 dias & 4 dias \\
\hline T. roseum $(\mathrm{F} 1)$ & $47 \mathrm{~b}$ & $93 \mathrm{a}$ & $25 \mathrm{~b}$ & 88 a \\
\hline T. roseum $(\mathrm{F} 2)$ & $60 \mathrm{~b}$ & $93 \mathrm{a}$ & $15 \mathrm{~b}$ & 83 a \\
\hline Cephalosporium sp (F3) & $87 \mathrm{a}$ & $100 \mathrm{a}$ & $43 \mathrm{a}$ & $93 \mathrm{a}$ \\
\hline T. roseum $(\mathrm{F} 4)$ & $63 \mathrm{~b}$ & $100 \mathrm{a}$ & $18 \mathrm{~b}$ & 83 a \\
\hline Penicillium sp (F9) & $60 \mathrm{~b}$ & $100 \mathrm{a}$ & $43 \mathrm{a}$ & 93 a \\
\hline Alternaria sp (F13) & $90 \mathrm{a}$ & $97 \mathrm{a}$ & $33 \mathrm{a}$ & 85 a \\
\hline Fosfito $\mathrm{Ca}(\mathrm{Ca} 6 \%)$ & $33 \mathrm{c}$ & $90 \mathrm{a}$ & $30 \mathrm{a}$ & 80 a \\
\hline Fosfito K (K2O 20\%) & $57 \mathrm{~b}$ & $97 \mathrm{a}$ & $28 \mathrm{a}$ & $68 \mathrm{a}$ \\
\hline Iprodione & $03 \mathrm{~d}$ & $13 \mathrm{~b}$ & $0 \mathrm{~b}$ & $3 \mathrm{~b}$ \\
\hline Testemunha & $63 \mathrm{~b}$ & $100 \mathrm{a}$ & $55 \mathrm{a}$ & $95 \mathrm{a}$ \\
\hline CV (\%) & 21,98 & 5,85 & 41,55 & 12,36 \\
\hline
\end{tabular}

${ }^{1}$ Médias de 3 repetições, cada uma constituída por 10 frutos, todos com 4 ferimentos.

${ }^{2}$ Dados originais. As médias seguidas pela mesma letra não diferem estatisticamente pelo teste de Scott-Knott a 5\% de significância.

Segundo Janisiewicz e Korsten (2002), para a podridão parda do pessegueiro, que pode se originar de infecções latentes no pomar ou ferimentos durante ou após a colheita, seriam necessárias estratégias de aplicação de antagonistas nos períodos de pré e pós-colheita para se alcançar um bom controle. Assim, seria possível reduzir significativamente os danos, pois, com a aplicação antes da colheita, permite-se que a população do antagonista se estabeleça na superfície dos frutos antecipadamente a qualquer ferimento provocado na colheita. E conforme Janisiewicz e Korsten (2002), caso estes sejam provocados na superfície dos frutos, os ferimentos são um ambiente ideal ao antagonista, porque são regiões providas de umidade, ricas em nutrientes e que não apresentam competição com outros microrganismos.

Atualmente, podem ser encontrados relatos do isolamento de diversos microrganismos, incluindo bactérias, fungos filamentosos e leveduras testados em frutos durante a pós-colheita no controle de patógenos (SCHENA et al. 2003). Alguns trabalhos mostraram a utilização das leveduras Cryptococcus spp. Skinner e Rhodotorula spp. Karl-Heinz no controle de $P$. expansum Link em maçãs; A. pullulans, Candida vanderwaltii Vidal-Leiria e C. oleophila Bennett em uva e kiwi (Actinidia chinensis Lindl.), contra Botrytis cinerea Pers.:Fr.; Trichoderma atroviride Karsten e T. viride Pers.:Fr. em pêssegos e ameixas, contra M. fructicola; Pseudomonas syringae van Hall em pêssegos, no controle de M. fructicola; e $A$. pullulans em cerejas, contra B. cinerea e M. laxa (Aderh. et Ruhl.) Honey (CHAND-GOYAL; SPOTTS, 1996; HONG et al. 1998; SCHENA et al. 2003). 
De acordo com os resultados deste trabalho, poderia ser indicada uma continuidade da pesquisa, com a utilização de misturas ou alternâncias dos antagonistas com fosfitos, concomitantemente à avaliação do efeito desses produtos no desenvolvimento dos microrganismos, pois, com essa medida, poder-se-ia ampliar o espectro de ação, intensificando a eficiência do controle.

\section{REFERÊNCIAS}

BONATERRA, A.; MARI, M.; CASALINI, L.; MONTESINOS, E. Biological control of Monilinia laxa and Rhizopus stolonifer in postharvest of stone fruit by Pantoea agglomerans EPS125 and putative mechanisms of antagonism. International Journal of Food Microbiology, Oxford, v. 84, p. 93-104, 2003.

BONETI, J. I.; KATSURAYAMA, Y. Viabilidade do uso de fosfitos no manejo das doenças da macieira. In: ENCONTRO NACIONAL SOBRE FRUTICULTURA DE CLIMA TEMPERADO, 5., 2002, Fraiburgo. Anais... Fraiburgo: Epagri, 2002. p.125-139.

CANTERI, M. G.; Althaus, R. A.; VIRGENS Filho, J. S.; GiGliOTi, E. A.; GODOY, C. V. SASM-Agri: Sistema para análise e separação de médias em experimentos agrícolas pelos métodos ScottKnott, Tukey e Duncan. Revista Brasileira de Agrocomputação, Ponta Grossa, v. 1, n. 2, p. 18-24, 2001.

CHAND-GOYAL, T.; SPOTTS, R. A. Postharvest biological control of blue mold of apple and brown rot of sweet cherry by natural saprophytic yeasts alone or in combination with low doses of fungicides. Biological Control, Orlando, v. 6, p. 253-259, 1996.

DALBÓ, M. A.; SCHUCK, E. Avaliação do uso de fosfitos para o controle do míldio da videira. Agropecuária Catarinense, Florianópolis, v. 16, n. 3, p. 33-36, 2003.

DE CAL, A.; SAGASTA, E, M.; MELGAREJO, P. Biological control of peach twig blight (Monilinia laxa) with Penicillium frequentans. Plant Pathology, London, v. 39, n. 4, p .612-618, 1990.

EMERY, K. M.; SCHERM, H.; SAVELLE, A. T. Assessment of interactions between components of fungicide mixtures against Monilinia fructicola. Crop Protection, Oxford, v. 21, p. 41-47, 2002.

HONG, C. X.; MICHAILIDES, T. J. Prune, plum, and nectarine as hosts of Trichothecium roseum in California orchards. Plant Disease, Saint Paul, v. 81, n .1, p. 112, 1997. Resumo.

HONG, C.; MICHAILIDES, T. J.; HOLTZ, B. A. Effects of wounding, inoculum density, and biological control agents on postharvest brown rot of stone fruits. Plant Disease, Saint Paul, v. 82, n. 11, p. 12101216, 1998.

IPPOLITO, A.; NIGRO, F. Impact of preharvest application of biological control agents on postharvest diseases of fresh fruits and vegetables. Crop Protection, Oxford, v. 19, p. 715-723, 2000.

JANISIEWICZ, W. J.; KORSTEN, L. Biological control of postharvest diseases of fruits. Annual Review of Phytopathology, Palo Alto, v. 40, p. 411-441, 2002.

LARENA, I.; TORRES, R.; DE CAL, A.; LIÑÁN, M.; MELGAREJO, P.; DOMENICHINI, P.; BELLINI, A.; MANDRIN, J. F.; LICHOU, J.; ERIBE, X. O. de; USALL, J. Biological control of postharvest Brown rot (Monilinia spp.) of peaches by field applications of Epicoccum nigrum. Biological Control, Orlando, v. 32, p. 305-310, 2005.

LUO, Y.; MICHAILIDES, T. J. Threshold conditions that lead latent infection to prune fruit rot caused by Monilinia fructicola. Phytopathology, Saint Paul, v. 93, n. 1, p. 102-111, 2003.

LUO, Y.; MORGAN, D. P.; MICHAILIDES, T. J. Risk analysis of rot blossom blight of prune caused by Monilinia fructicola. Phytopathology, St. Paul, v. 91, n 8, p. 759-768, 2001.

MADRIGAL, C.; PASCUAL, S.; MELGAREJO, P. Biological control of peach twig blight (Monilinia laxa) with Epicoccum nigrum. Plant Pathology, London, v. 43, p. 554-561, 1994. 
MAY-DE MIO, L. L.; MONTEIRO, L. B.; DE NAZARENO, N. R. X.; HICKEL, E. Classificação e manejo dos agroquímicos em fruteiras de caroço. In: MONTEIRO, L. B.; MAY-DE MIO, L. L.; SERRAT, B. M.; MOTTA, A. C.; CUQUEL, F. L. Fruteiras de caroço: uma visão ecológica. Curitiba: Universidade Federal do Paraná, 2004. p. 263-297.

MOREIRA, L. M. Controle químico e biológico de Monilinia fructicola (Wint) Honey e monitoramento de infecções latentes em frutos. 76f. Dissertação (Mestrado em Produção Vegetal) Setor de Ciências Agrárias, Universidade Federal do Paraná, Curitiba, 1999.

MOREIRA, L. M.; MAY-DE MIO, L. L.; VALDEBENITO-SANHUEZA, R. M.; LIMA, M. L. R. Z. C; POSSAMAI, J. C. Controle em pós-colheita de Monilinia fructicola em pêssegos. Fitopatologia Brasileira, Brasília, v. 27, n. 4, p. 395-398, 2002.

SCHENA, L.; NIGRO, F.; PENTIMONE, I.; LIGORIO, A.; IPPOLITO, A. Control of postharvest rots of sweet cherries and table grapes with endophytic isolates of Aureobasidium pullulans. Postharvest Biology and Technology, Oxford, GB, v. 30, p. 209-220, 2003.

SÔNEGO, O. R.; GARRIDO, L. da R.; CZERMAINSKI, A. B. C. Avaliação do fosfito de potássio no controle do míldio da videira. Bento Gonçalves: EMBRAPA, 2003. 14p. (Boletim de Pesquisa e Desenvolvimento, n. 11).

WADT, L.; NOGUEIRA, E. M. de C.; CORRENTE, J. E. Controle químico da podridão parda (Monilinia fructicola) em nectarina (Prunus persica var. nucipersica). Arquivos do Instituto Biológico, São Paulo, v. 66, n. 1, p. 9-14, 1999.

WITTIG, H. P. P.; JOHNSON, K. B.; PSCHEIDT, J. W. Effect of epiphytic fungi on brown rot blossom blight and latent infections in sweet cherry. Plant Disease, Sain Paul, v. 81, n. 4, p. 383-387, 1997. 